\title{
Performance Evaluation of DYMO, AODV and DSR Routing Protocols in MANET
}

\author{
Muneer Alshowkan \\ Department of Computer \\ Science \& Engineering, \\ University of Bridgeport \\ 126 Park Avenue
}

\author{
Eman Abdel Fattah \\ Department of Computer \\ Science \& Engineering, \\ University of Bridgeport \\ 126 Park Avenue
}

\author{
Ammar Odeh \\ Department of Computer \\ Science \& Engineering, \\ University of Bridgeport \\ 126 Park Avenue
}

\begin{abstract}
An Ad Hoc network has no infrastructure and it provides flexibility to network's nodes to connect and disconnect at any time. A MANET is an Ad Hoc network that provides freedom of movement to the network's nodes. In a MANET, The development of routing protocols is one of the main challenges and much research has been done to evaluate the current protocols to improve them or to develop new protocols. There are several protocols that have been tested in the past and each protocol has its advantages and disadvantages depending on the applications. In this paper, we evaluate Dynamic MANET On-demand routing protocol (DYMO), Ad Hoc On-Demand Distance Vector Routing protocol (AODV) and Dynamic Source Routing protocol (DSR). We evaluate the performance based on throughput, dropped packets and end-to-end delay.
\end{abstract}

\section{General Terms}

Mobile Communication, Wireless Networks

\section{Keywords}

DYMO, Ad Hoc, MANET, NS2, DSR, AODV, Simulation, Wireless, Routing protocol

\section{INTRODUCTION}

A MANET is an ad Hoc network. It is a decentralized network where there are several nodes interacting with one another without the existence of a network infrastructure as shown in Figure 1. All the nodes in the ad-hoc network are temporary and are gathered together randomly. In infrastructure networks, the access point is responsible for the nodes' connectivity and the distribution of the packets among the nodes in the network. In a MANET, each node can send and receive packets. Also, a node can act as a router to route packets between two or more nodes to keep the connectivity among them. The route from one node to another goes through the neighbor nodes if there is no direct route available. Figure 2 shows the classification of the routing protocols in a MANET. There are two categories; proactive (Table driven) protocols and reactive (On-Demand) protocols. Next two subsections introduce a brief summary about proactive protocols and reactive protocols.

\subsection{Proactive Protocol}

Proactive protocols of Ad Hoc networks are derived from distance vector and link state protocols which are designed for wired network. The main idea of these protocols is to periodically update routing tables through exchange of routes' updates among the network's nodes. The advantage of these protocols is the instant availability of routes whenever they are needed. However, it has a disadvantage of the large overhead to maintain these routing tables.

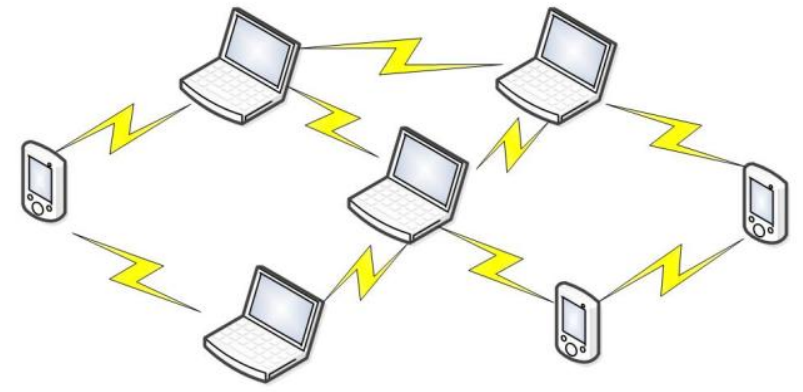

Figure 1: An Example of Ad Hoc Network

\subsection{Reactive Protocols}

Reactive protocols are different from proactive protocols because they aim to reduce the overhead involved in proactive protocols. The route is maintained only when it is needed. Such an approach does not require to periodically maintaining the routing tables. The routes are updated when there is a need to use them. In reactive protocols, a route is discovered only when there is data to transmit. Ad Hoc On-Demand Distance Vector protocol (AODV), Dynamic Source Routing protocol (DSR), and Dynamic MANET On-demand protocol (DYMO) are classified as reactive routing protocols. A brief description about each of these protocols is presented in the following subsections.

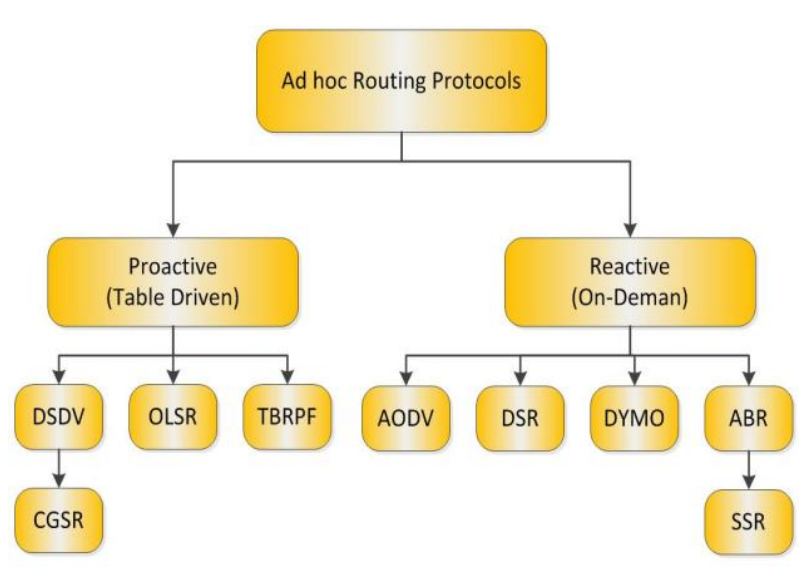

Figure 2: Ad Hoc Routing Protocol List

1.2.1 Ad Hoc On-Demand Distance Vector

Ad Hoc On-Demand Distance Vector (AODV) is a reactive 
ad-hoc routing protocol. In this protocol, there are two main operations which are the route discovery and the route maintenance. Also, AODV has four types of messages; Route Request (RREQ), Route Reply (RREP), Route Error (RERR) and HELLO (to monitor the link status). AODV broadcasts RREQ messages only when it needs to discover a path to the destination. When the message is propagated in the network, the nodes use it to update their routing tables by the information that the message contains. The message contains the sequence number of the final destination which should be greater than or equal to that node's number. The destination node or any node that has a fresh an updated rout to the target node replies with RREP message. A node issues RERR message to update broken routes with the neighboring nodes. Hello message is used to check the connectivity with the other nodes. Also, Hello message is used with Time to Live (TTL). If there is no reply received in the TTL, then the nodes are considered as lost nodes.

\subsubsection{Dynamic Source Routing}

A Dynamic Source Routing (DSR) is a reactive ad-hoc routing protocol and has two main operations; one is route discovery and the other one is route maintenance. Route discovery is used by a node to get the route to another node. The node initiates a RREQ message that floods the network. A RREQ message contains unique ID, the source, and the destination addresses. When an intermediate node receives the RREQ message, it appends its address and forwards it again. When the destination node receives the RREQ, it initiates a RREP message and sends it back to the originator using the route in the RREQ message. When the source receives the RREP message from the destination, it caches the route in its route cache. In the route maintenance operation, this protocol requires the next hop to acknowledge receiving the packet. If there is a breakage in the route, the source node will get a RERR packet indicating the link is broken. The source node can use another route in the route cache or perform new routing discovery.

\subsubsection{Dynamic MANET On-demand}

A Dynamic MANET On-demand (DYMO) is a reactive adhoc routing protocol. It has two main operations; one is route discovery and the other one is route maintenance. The node only discovers the route to the destination when it has packets to send. In this case it updates its table with the destination node's route. The source node floods message RREQ into the network. When an intermediate node receives the RREQ, it records the address to the source node of the RREQ and forwards the message to the neighboring nodes. When the final destination receives the RREQ message with its address as a new destination, it initiates a route reply message RREP with the source node as destination and unicasts that message in the network. The intermediate nodes update their routing tables and forward the RREP message to the destination. The route maintenance occurs when the route to a specific node is broken and there are packets to send to the node at the end of the broken route.

\section{MOTIVATION}

A MANET provides and manages connectivity between network's nodes regardless of the location and the geographical positions of these nodes. The flexibility of a MANET makes it attractive and promising as MANET is selfconfiguring and it doesn't need infrastructure to establish a connection between the network's nodes. But, there are challenges in this type of network. As the network's nodes move, there is a need to update the nodes with the current positions of other nodes and the best way to contact them.
The transmission range of the nodes is limited as shown in Figure 3. Also, when the network's geographical size increases, the latency also increases. Moreover, the routing from one node to another node depends on the routing protocol which plays an essential role in a MANET performance. Due to the impact of the routing protocols on MANETs performance, this paper aims to provide an extensive evaluation of the performance for AODV, DSR and DYMO routing protocols in a MANET.

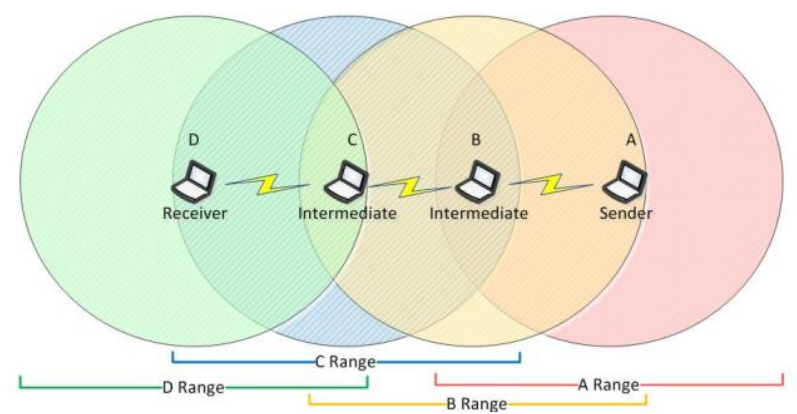

Figure 3: Nodes Ranges and Routing

\section{RELATED WORK}

In the recent years many researchers focus on developing efficient algorithms for wireless networks. Ad Hoc networks have been one of the promising areas of research due to their flexibility and the different applications they support. However, it is still facing several challenges that are under investigation and evaluation. MANET networks are part of ad Hoc networks where the mobility of the nodes provides further challenges.

Performance evaluation studies of MANETs have been carried out by several researchers. In [1], the authors evaluate three ad-hoc routing protocols under MANET. The evaluation has been done comparing two reactive routing protocols; AODV and DSR and one proactive routing protocol; DSDV. An evaluation study based on the effect of nodes' speed, effect of TCP protocol types and the effect of maximum length of the queue is performed. DSR shows better performance than AODV and DSDV in the steady state. AODV shows better performance in TCP traffic. DSDV performs better when it comes to the maximum length of the queue.

Asma et. al. evaluate three ad-hoc routing protocols; DSDV, AODV and DSR [2]. The DSDV has low throughput and high routing load compared to AODV and DSR. AODV and DSR perform very well and while in some situations the AODV performs better. The overall performance of DSR exceeds the other two protocols based on the average end-toend delay. Moreover, changing the packet size doesn't affect the DSDV performance but it affects the performance of both AODV and DSR protocols. However, all protocols perform well with respect to low mobility networks.

In the Monarch Project [3], the authors make a comprehensive study about the following routing protocols; AODV, DSDV, TORA, and DSR. The study is about the periodic advertisements, On-demand route-discovery, hop-byhop routing, source routing, and the used of the feedback from the MAC layer when there is a failure. The authors conclude that the DSR is performing better than the other routing protocols at different mobility factors such as the mobility rate and the movement speeds.

Sagar et. al. compare two routing protocols; Dynamic MANET On-demand (DYMO) as a reactive protocol, and 
Optimized Link State Routing (OLSR) as a proactive protocol [4]. The comparison is with respect to the packet delivery ratio, average end-to-end delay and the normalized routing overhead. The authors conclude that the performance of DYMO is better than OLSR in both MANET and Vehicle Ad Hoc Network (VANET).

In [5], a study of DYMO routing protocol with respect to average end-to-end throughput, average end-to-end delay, the routing overhead, the packet delivery ratio, and path optimality is conducted. The authors compare AODV and DYMO routing protocols and they conclude that DYMO routing protocol outperforms AODV because it is able to handle different mobility ranges and various traffic patterns.

Bisoyi et. al. evaluate three reactive routing protocols; DYMO, AODV and DSR [6]. The performance measured the throughput, packet loss, latency, packet delivery ratio and normalized routing load is examined. The evaluation is studied based on different factors for each routing protocol and based on the pause time. The author found that DYMO performance was better than the other routing protocols in the Quality of Service, throughput, and packet delivery ratio, delay, and normalized overhead.

\section{PROTOCOL SIMULATION}

The simulation environment is the network simulator NS2. It's built in $\mathrm{C}++$ and its interface uses OTcl which is an object oriented extension of Tool Command Language (Tcl). NS2 is popular in simulating routing protocols especially ad Hoc networks. It's also used in simulation of wired networks. There are several versions of network simulator NS2 and each version provides some improvements. NS2 allows the users to modify the source file and customize it. Also, NS2 allows the users to add new protocols to use them in the simulation. Linux Ubuntu 11.10 operating system is used as the operating system under the network simulator. The default parameters for each routing protocol are used. Table 1 shows the used parameters in the simulation.

\section{PERFORMANCE EVALUATION AND METRICS}

We used the following metrics to evaluate and test the performance among the three routing protocols. The throughput, the dropped packets and the end-to-end delay are examined.

\subsection{Throughput of receiving packets}

This parameter measures the successful packets received at the final destination that are sent from the sender.

\subsection{Dropped packets}

This parameter is used to discover the dropped packets between two nodes.

\subsection{End-to-End Delay}

This parameter determines the delay of the sent packets between the first node which is the sender and the second node which is the receiver across the MANET network.

Table 1: Simulation Parameters in NS2

\begin{tabular}{|l|c|}
\hline \multicolumn{1}{|c|}{ Parameter } & Value \\
\hline NS-2 Version & 2.35 \\
\hline Channel Type & Wireless Channel \\
\hline Radio-Propagation & TwoRayGround \\
\hline Network Interface & WirelessPhy \\
\hline
\end{tabular}

\begin{tabular}{|l|c|}
\hline MAC Type & IEEE 802.11 \\
\hline Antenna & OmniAntenna \\
\hline Max Packet in Queue & 50 \\
\hline Number of Nodes & $700 \times 700$ \\
\hline Area & $160 \mathrm{sec}$ \\
\hline Simulation Time & UDP \\
\hline Data Type & 1000 bytes \\
\hline Packet Size & $3,5,7,10,15,20,25$ \\
\hline Nodes' Speed & \\
\hline
\end{tabular}

\section{SIMULATION RESULTS}

\subsection{Throughput of receiving packets}

Figure 4 shows the throughput of receiving packets in AODV routing protocol. It increases till around 500 packets / TIL (Time Interval Length) then it stays stable until 70 seconds then it starts to drop.

The DSR throughput of receiving packets is shown in Figure 5. It increases to 500 packets / TIL then it starts to drop and increases again due to the change in the network and because of the nodes' movement. At around 60 seconds of the simulation time, the throughput drops again until around 80 seconds. Then, it gets better in the range from 100 to 120 seconds then it starts to drop till the end of the simulation. DYMO throughput of receiving packets is shown in Figure 6. It increases to almost 490 packets / TIL and it stays stable for a while until it gets changed in the middle of the simulation. Then, it increases again to around 465 packets / TIL.

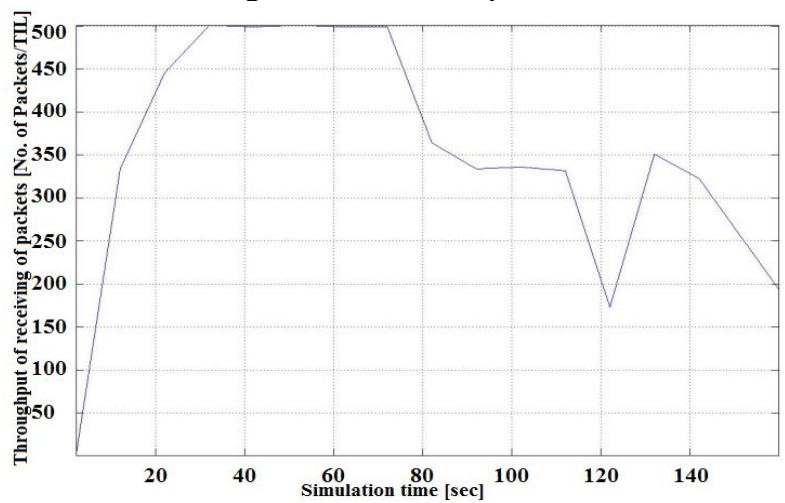

Figure 4: Throughput of Receiving Packets in AODV



Figure 5: Throughput of Receiving Packets in DSR 


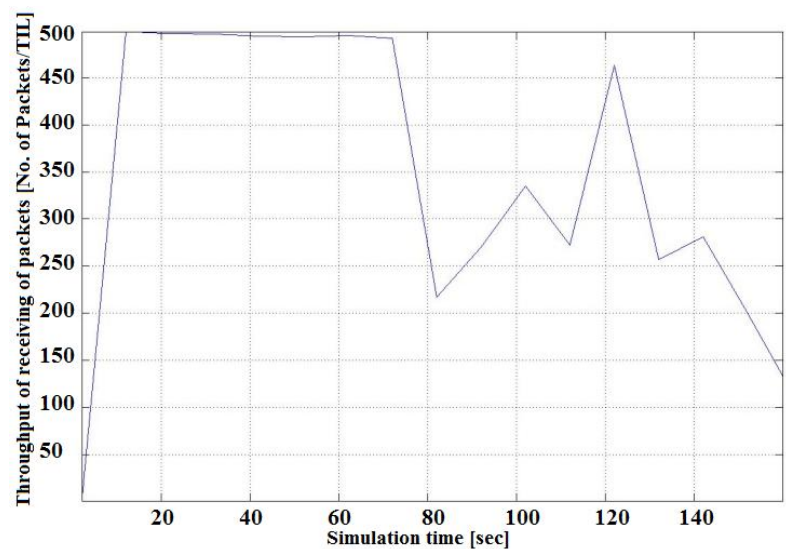

Figure 6: Throughput of Receiving Packets in DYMO

\subsection{Dropped Packets}

Figure 7 shows the AODV dropped packets. At the beginning of the simulation, there was high dropped packet and after that it stays stable for a while until around 77 seconds and after that it goes up again and ends up to cumulative 1850 dropped packets from the 6508 sent packets.

In DSR protocol, it doesn't face any dropping at the beginning then it starts to drop a little bit and after that it stables as shown in Figure 8. At around 70 seconds, the dropping starts and goes up until around 1890 packets from the 7546 sent packets.

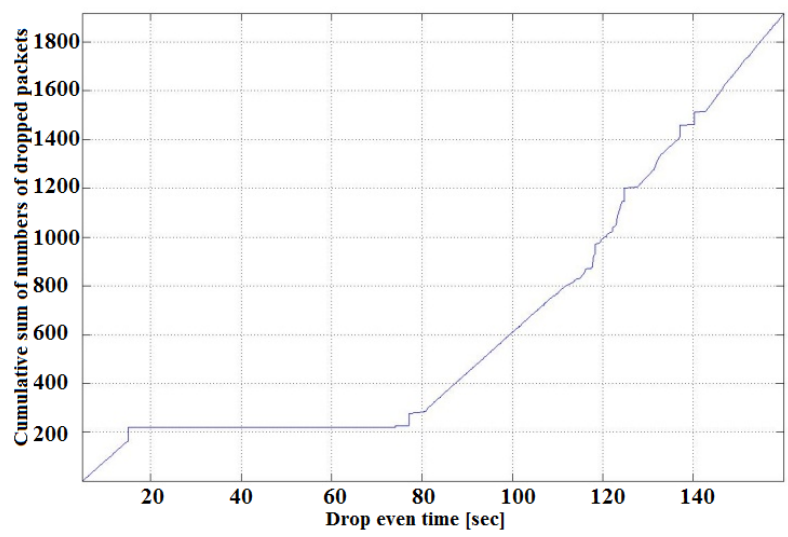

Figure 7: AODV Cumulative Sum of Dropped Packets



Figure 8: Cumulative Sum of Dropped Packets in DSR

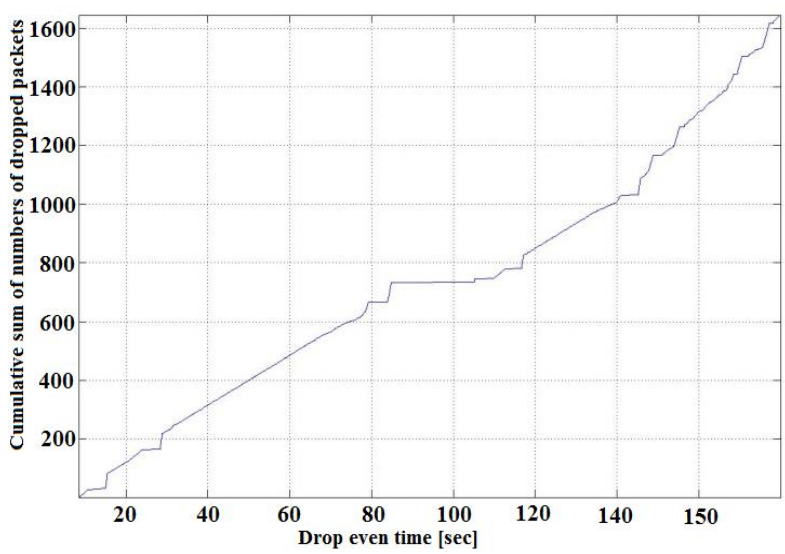

Figure 9: DYMO Cumulative Sum of Dropped Packets

Figure 9 shows the dropped packets in DYMO. The dropping starts slowly and goes to around 780 packets at around 110 seconds and it stays stable until around 122 seconds. Then it goes up to around 1620 dropped packets from the 9567 sent packets. When we compare the ratio of dropped packets to the sent packets in DYMO, we find that the ratio is less than other protocols.

\subsection{End-To-End Delay}

Figure 10 shows the end-to-end delay in AODV. When the simulation starts there is a delay, and then the delay drops and remains stable until around 80 seconds then the delay increases again between 120 and 140 seconds where the network faces large delay. The delay lasts for 14 seconds which is a large duration of time. After that the delay decreases and the simulation ends with a delay of around 4 seconds.

In DSR protocol, end-to-end delay is shown in Figure 11. There is a point of time where the delay goes up to around 12.5 seconds. However, the delay is less than 4 seconds in most of the simulation time.

Figure 12 shows end-to-end delay in DYMO protocol. The delay does not exceed 3 seconds and that occurs at around 80 seconds of the simulation time. Therefore, DYMO end-to-end delay is less than both AODV and DSR protocols. We will analyze the reason for this better performance in the next section.

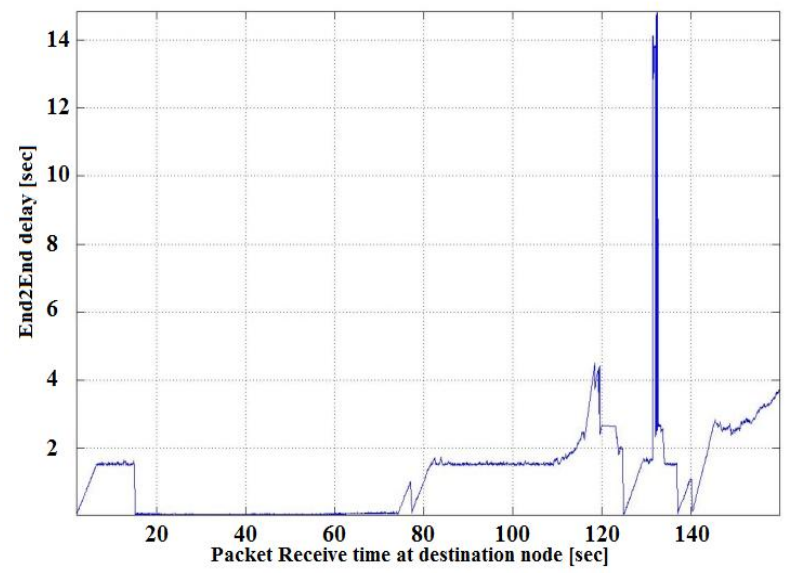

Figure 10: AODV End-To-End Delay 




Figure 11: DSR End-To-End Delay

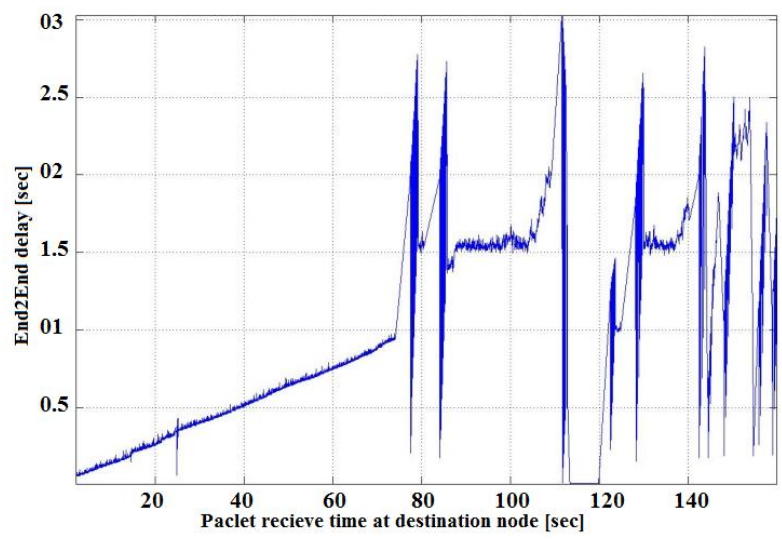

Figure 12: DYMO End-To-End Delay

\section{SIMULATION ANALYSIS}

The performance among the three protocols in the throughput of receiving packets is comparable. However, DYMO outperforms the other two protocols in terms of the dropped packets and end-to-end delay. DYMO uses a Management Information Base (MIB) which explains the improved performance over DSR and AODV. MIB is a database that contains objects which can manage the network devices and interfaces. Also, DYMO requires that the neighbor nodes to have full duplex links to improve the communications among the nodes Another advantage of DYMO is using Type Length Value (TLV) which is fast in searching using the generalized parsing function. Also, TLV allows the old messages which were received at the old router to be skipped and retransmitted using the correct route. Moreover, TLV allows the parsing in binary format for fast processing and searching [7].

\section{CONCLUSION}

In this paper, we evaluate the performance of three routing protocols; AODV, DSR and DYMO from three different aspects. For the first metric which is the throughput of receiving packets, the performance of the three protocols is comparable. With respect to the dropped packets and the endto-end delay, DYMO outperforms AODV and DSR. DYMO uses different and enhanced mechanisms compared to AODV and DSR protocols. DYMO has the ability to improve the routes according to the network behavior and it uses MIB and TLV, which allow DYMO to have better performance.

\section{REFERENCES}

[1] E. Abdelfattah, G. Liu, "Performance Evaluation of Mobile Ad-Hoc Routing Protocols," in International Joint Conferences on Computer, Information, and Systems Sciences, and Engineering", Bridgeport, CT, 2008.

[2] T. Asma, G. Rajneesh, T. Sunil, "Comparative Performance Analysis of DSDV, AODV and DSR Routing Protocols in MANET using NS2," in International Conference on Advances in Computer Engineering, Bangalore, Karnataka, India, 2010, 330.

[3]J. Broch, D. A. Maltz, D. B. Johnson, Y. Hu, J. Jetcheva, "A Performance Comparison of Multi-Hop Wireless Ad Hoc Network Routing Protocols" in Fourth Annual ACM/IEEE International Conference on Mobile Computing and Networking, Ballas, Texas, 1998.

[4] S. Sagar, J. Saqib, A. Bibi, N. Javaid, "Evaluating and Comparing the Performance of DYMO and OLSR in MANETs and in VANETs," in Multitopic Conference (INMIC), 2011 IEEE 14th International, Karachi, Pakistan, 2011, 362-366.

[5] M. Quan-xing, X. Lei, "DYMO Routing Protocol Research and Simulation Based on NS2,", in International Conference on Computer Application and System Modeling, Taiyuan, Shanxi, China, 2010, V1441.

[6] S. Bisoyi, S. Sahu, "Performance analysis of Dynamic MANET On-demand (DYMO) Routing protocol," in International Conference On Advances In Computer, Communication Technology \& Applications, Orissa, India, 2010.

[7] I. Chakeres, C. Perkins, Dynamic MANET On-demand (DYMO) Routing draft-ietf-manet-dymo-05, Mobile Ad hoc Networks Working Group, Nokia, June 20, 2006. 